\title{
NEW REFINEMENTS OF CUSA-HUYGENS INEQUALITY
}

\author{
CHRISTOPHE CHESNEAU, MARKO KOSTIĆ, BRANKO MALEŠEVIĆ, \\ BOJAN BANJAC, AND YOGESH J. BAGUL
}

\begin{abstract}
In the paper, we refine and extend Cusa-Huygens inequality by simple functions. In particular, we determine sharp bounds for $\sin (x) / x$ of the form $(2+\cos (x)) / 3-(2 / 3-2 / \pi) \Upsilon(x)$, where $\Upsilon(x)>0$ for $x \in$ $(0, \pi / 2), \Upsilon(0)=0$ and $\Upsilon(\pi / 2)=1$, such that $\sin x / x$ and the proposed bounds coincide at $x=0$ and $x=\pi / 2$. The hierarchy of the obtained bounds is discussed, along with a graphical study. Also, alternative proofs of the main result are given.
\end{abstract}

\section{INTRODUCTION}

The Cusa-Huygens inequality $[13,16]$ is one of the celebrated inequalities in the theory of analytic inequalities involving trigonometric functions. It is stated as follows:

$$
\frac{\sin x}{x}<\frac{2+\cos x}{3}, \quad x \in(0, \pi / 2)
$$

In the recent paper [22, Remark 4.1], it is remarked that the inequality (1.1) is, in fact, true for all $x \neq 0$. Due to the symmetry of the functions involved at both sides of (1.1), it suffices to consider the inequality on the right half of the real line. Although (1.1) is true for $x>0$ and it is not sharp everywhere. So the interest among researchers has been to consider the inequality in the natural intervals $(0, \pi / 2)$ or $(0, \pi)$. In [22], L. Zhu obtained Cusa-Huygens type inequalities on a wider range $(0, \pi)$. We must emphasize here that the sharp Cusa-Huygens type inequalities on a wider range $(0, \pi)$ have also appeared in $[5,10,15,17]$.

In $[8,20]$, the following Cusa-Huygens type double inequality was established:

$$
\left(\frac{2+\cos x}{3}\right)^{\alpha}<\frac{\sin x}{x}<\left(\frac{2+\cos x}{3}\right)^{\zeta}, \quad x \in(0, \pi / 2)
$$

2010 Mathematics Subject Classification. 26A48, 26D05, 33B10.

Key words and phrases. Cusa-Huygens inequality, trigonometric functions, l'Hospital's rule of monotonicity. 
where $\alpha=\ln (\pi / 2) / \ln (3 / 2) \approx 1.11374$ and $\zeta=1$ are the best possible constants. Simple alternative proofs of $(1.2)$ are offered in [3,4]. For other details about inequalities (1.1) and (1.2), we refer readers to [3-10,13-18, 20-22].

The main objective of this paper is to provide a manageable and sharp alternative to the double inequality (1.2) in the following sense: (i) in the applications, the exponentiated version of $(2+\cos (x)) / 3$ is not always manageable, so we have in mind to keep the overall simplicity of the former Cusa-Huygens inequality by taking the unit exponent, and (ii) the values of the bounds in (1.2) differ to the one of $\sin x / x$ when $x=\pi / 2$, making them not sharp for an interval of values of $x$ close to $\pi / 2$. For these reasons, we focus our attention on sharp bounds of $\sin x / x$ of the following form:

$$
Z(x):=\frac{2+\cos x}{3}-\left(\frac{2}{3}-\frac{2}{\pi}\right) \Upsilon(x),
$$

where $\Upsilon(x)>0$ for $x \in(0, \pi / 2), \Upsilon(0)=0$ and $\Upsilon(\pi / 2)=1$. Hence, a simple

form for $\Upsilon(x)$ implies a tractable expression for $Z(x)$. Candidates of such functions are proposed and two theorems are proved. The importance of the finding is illustrated by a graphical study, showing the high degree of sharpness of the results. Also, the hierarchy between the obtained bounds are examined. Alternative proofs are also provided.

The rest of the paper is divided into the following sections. Section 2 contains the main results. Section 3 is devoted to the proofs of these results. Diverse complements are offered in Section 4, including a comparison of the proposed bounds, a graphical study and alternative proofs.

\section{MAin RESUlts}

The following theorem presents sharp bounds for $\sin x / x$ of the form (1.3), with $\Upsilon(x)$ defined as exponentiated version of $(\pi / 2-1)^{-1}(x-\sin x)$.

Theorem 1. Let $x \in(0, \pi / 2)$. Then the double inequality

$$
\frac{2+\cos x}{3}-\left(\frac{2}{3}-\frac{2}{\pi}\right) \Phi_{1}(x)<\frac{\sin x}{x}<\frac{2+\cos x}{3}-\left(\frac{2}{3}-\frac{2}{\pi}\right) \Phi_{2}(x)
$$

where $\Phi_{1}(x):=(\pi / 2-1)^{-1}(x-\sin x)$ and $\Phi_{2}(x):=(\pi / 2-1)^{-2}(x-\sin x)^{2}$.

In a similar fashion to Theorem 1, Theorem 2 presents sharp bounds for $\sin x / x$ of the form (1.3), with $\Upsilon(x)$ defined as exponentiated version of $\sin x-$ $x \cos x$.

Theorem 2. Let $x \in(0, \pi / 2)$. Then, the double inequality

$$
\frac{2+\cos x}{3}-\left(\frac{2}{3}-\frac{2}{\pi}\right) \Psi_{1}(x)<\frac{\sin x}{x}<\frac{2+\cos x}{3}-\left(\frac{2}{3}-\frac{2}{\pi}\right) \Psi_{2}(x)
$$

where $\Psi_{1}(x):=\sin x-x \cos x$ and $\Psi_{2}(x):=(\sin x-x \cos x)^{2}$. 
Since $\Psi_{2}(x)$ and $\Psi_{2}(x)$ are positive, the Cusa-Huygens inequality in (1.1) is clearly refined. Moreover, as linear combinations of simple functions, the obtained bounds are quite manageable for diverse analytical manipulations involving integration, series, and so on.

The main proofs of these theorems are based on several general and specific results described in the next section. Some of them can be of independent interest, for aims beyond the scope of this study. Let us mention that the sharpness of the obtained bounds, as well as alternative proofs, will be discussed later.

\section{Proofs}

3.1. Preliminaries and lemmas. The following series expansions can be found in $[11,1.411(7,11)]$ :

$$
\cot x=\frac{1}{x}-\sum_{k=1}^{\infty} \frac{2^{2 k}\left|B_{2 k}\right|}{(2 k) !} x^{2 k-1}, \quad x \in(0, \pi)
$$

and

$$
\frac{1}{\sin x}=\frac{1}{x}+\sum_{k=1}^{\infty} \frac{2\left(2^{2 k-1}-1\right)\left|B_{2 k}\right|}{(2 k) !} x^{2 k-1}, \quad x \in(0, \pi) .
$$

From (3.2), we can write

$$
\frac{x}{\sin x}=1+\sum_{k=1}^{\infty} \frac{2\left(2^{2 k-1}-1\right)\left|B_{2 k}\right|}{(2 k) !} x^{2 k}, \quad x \in(0, \pi) .
$$

Similarly, we obtain

$$
\left(\frac{x}{\sin x}\right)^{2}=-x^{2}(\cot x)^{\prime}=1+\sum_{k=1}^{\infty} \frac{2^{2 k}(2 k-1)\left|B_{2 k}\right|}{(2 k) !} x^{2 k}, \quad x \in(0, \pi)
$$

and

$$
\frac{\cos x}{\sin ^{2} x}=\left(\frac{1}{\sin x}\right)^{\prime}=\frac{1}{x^{2}}-\sum_{k=1}^{\infty} \frac{(2 k-1)\left(2^{2 k}-2\right)\left|B_{2 k}\right|}{(2 k) !} x^{2 k-2}, \quad x \in(0, \pi),
$$

respectively, from (3.1) and (3.2).

For Lemma 1, we refer to [2]. Lemma 1 is known as l'Hôpital's rule of monotonicity.

Lemma 1. Let $f, g:[m, n] \rightarrow \mathbb{R}$ be two continuous functions which are differentiable on $(m, n)$ and $g^{\prime}(x) \neq 0$ in $(m, n)$. If $f^{\prime}(x) / g^{\prime}(x)$ is increasing (or decreasing) on $(m, n)$, then the functions $(f(x)-f(m)) /(g(x)-g(m))$ and $(f(x)-f(n)) /(g(x)-g(n))$ are also increasing (or decreasing) on $(m, n)$. If 
$f^{\prime}(x) / g^{\prime}(x)$ is strictly monotone, then the monotonicity in the conclusion is also strict.

Lemma 2. ( [6, Lemma 2]) The function

$$
P(x):=\frac{x^{2} \sin x}{\sin x-x \cos x}, \quad x \in(0, \pi / 2)
$$

is positive and strictly decreasing on $(0, \pi / 2)$.

Lemma 3 below can be found in $[1,12]$.

Lemma 3. Let $A(x):=\sum_{n=0}^{\infty} a_{n} x^{n}$ and $B(x):=\sum_{n=0}^{\infty} b_{n} x^{n}$ be convergent for $|x|<T$, where $a_{n}$ and $b_{n}$ are real numbers for $n=0,1,2, \cdots$ such that $b_{n}>0$, and $T>0$ is a fixed constant. If the sequence $a_{n} / b_{n}$ is strictly increasing(or decreasing), then the function $A(x) / B(x)$ is also strictly increasing(or decreasing) on $(0, T)$.

The next lemma is about lower and upper bounds for a ratio involving absolute Bernoulli numbers. It is established in [19].

Lemma 4. For $k \in \mathbb{N}$, the Bernoulli numbers satisfy

$$
\frac{\left(2^{2 k-1}-1\right)}{\left(2^{2 k+1}-1\right)} \frac{(2 k+1)(2 k+2)}{\pi^{2}}<\frac{\left|B_{2 k+2}\right|}{\left|B_{2 k}\right|}<\frac{\left(2^{2 k}-1\right)}{\left(2^{2 k+2}-1\right)} \frac{(2 k+1)(2 k+2)}{\pi^{2}} .
$$

The following result is for future technical considerations.

Lemma 5. The function

$$
Q(x):=\frac{3 \sin x-2 x-x \cos x}{x^{5}}, \quad x \in(0, \pi / 2)
$$

is negative and strictly increasing on $(0, \pi / 2)$.

Proof. First, $Q(x)$ is negative due to inequality (1.1). Now, let

$$
\begin{aligned}
q_{1}(x):=3 \sin x-2 x-x \cos x, & h_{1}(x):=x^{5}, \\
q_{2}(x):=x \sin x+2 \cos x-2, & h_{2}(x):=5 x^{4}
\end{aligned}
$$

and

$$
q_{3}(x):=x \cos x-\sin x, h_{3}(x):=20 x^{3} .
$$

Then

$$
q_{i}(0+)=h_{i}(0+)=0(i=1,2,3), \quad \frac{q_{i}^{\prime}(x)}{h_{i}^{\prime}(x)}=\frac{q_{i+1}(x)}{h_{i+1}(x)}(i=1,2)
$$

and

$$
\frac{q_{3}^{\prime}(x)}{h_{3}^{\prime}(x)}=-\frac{1}{60} \frac{\sin x}{x} .
$$

Since the function $\sin x / x$ is strictly decreasing on $(0, \pi / 2)$, repeated application of Lemma 1 gives that $Q(x)$ is strictly increasing on $(0, \pi / 2)$. 
The following result is also an important ingredient of a main proof.

Lemma 6. The function

$$
T(x):=\frac{x^{2} \sin x}{x-\sin x}, \quad x \in(0, \pi)
$$

is positive and strictly decreasing on $(0, \pi)$.

Proof. Clearly, $T(x)$ is positive since $x>\sin x, x \in(0, \pi)$. Thus, it suffices to prove that $1 / T(x)$ is strictly increasing on $(0, \pi)$. We have

$$
\frac{1}{T(x)}=\frac{1}{x^{2}}\left(\frac{x}{\sin x}-1\right) \text {. }
$$

By (3.3), we get

$$
\frac{1}{T(x)}=\sum_{k=1}^{\infty} \frac{2\left(2^{2 k-1}-1\right)\left|B_{2 k}\right|}{(2 k) !} x^{2 k-2}, \quad x \in(0, \pi)
$$

which is obviously strictly increasing on $(0, \pi)$.

\subsection{Proofs of main results.}

Proof of Theorem 1. We first prove the left inequality of (2.1). Let us set

$$
f(x):=\frac{\frac{\sin x}{x}-\frac{2+\cos x}{3}}{x-\sin x}=\frac{3 \sin x-x \cos x-2 x}{3 x^{2}-3 x \sin x}, \quad x \in(0, \pi / 2) .
$$

As intermediary functions, we consider

$$
\begin{gathered}
g_{1}(x):=3 \sin x-x \cos x-2 x, \quad h_{1}(x):=3 x^{2}-3 x \sin x, \\
g_{2}(x):=x \sin x+2 \cos x-2, \quad h_{2}(x):=6 x-3 \sin x-3 x \cos x,
\end{gathered}
$$

and

$$
g_{3}(x):=x \cos x-\sin x, \quad h_{3}(x):=3 x \sin x-6 \cos x+6 .
$$

Then

$$
g_{i}(0+)=h_{i}(0+)=0(i=1,2,3), \quad \frac{g_{i}^{\prime}(x)}{h_{i}^{\prime}(x)}=\frac{g_{i+1}(x)}{h_{i+1}(x)}(i=1,2)
$$

and

with

$$
\frac{g_{3}^{\prime}(x)}{h_{3}^{\prime}(x)}=\frac{-x \sin x}{9 \sin x+3 x \sin x}=-\frac{1}{3}\left(\frac{x}{3+u(x)}\right)
$$

$$
u(x):=\frac{x}{\tan x} .
$$

The function $u(x)$ is positive and strictly decreasing on $(0, \pi / 2)$. Applying Lemma 1 repeatedly, we get that $f(x)$ is strictly decreasing on $(0, \pi / 2)$. Hence, for $x<\pi / 2$, we have

$$
f\left(\frac{\pi}{2}-\right)<f(x)
$$


Since

$$
f\left(\frac{\pi}{2}-\right)=\frac{\frac{2}{\pi}-\frac{2}{3}}{\frac{\pi}{2}-1}
$$

we get required left inequality of (2.1).

Now we prove the right inequality of (2.1). Let

$$
F(x):=\frac{\frac{\sin x}{x}-\frac{2+\cos x}{3}}{(x-\sin x)^{2}}=\frac{3 \sin x-x \cos x-2 x}{3 x^{3}-6 x^{2} \sin x+3 x \sin ^{2} x} .
$$

It can also be written as

$$
F(x)=\frac{3 \frac{1}{\sin x}-\frac{2}{x}\left(\frac{x}{\sin x}\right)^{2}-x \frac{\cos x}{\sin ^{2} x}}{3 x\left(\frac{x}{\sin x}\right)^{2}-6 x\left(\frac{x}{\sin x}\right)+3 x} .
$$

Utilizing (3.2), (3.3), (3.4) and (3.5), we can write

$F(x)=$

$$
\begin{aligned}
& \left(\frac{\sum_{k=1}^{\infty} \frac{6\left(2^{2 k-1}-1\right)}{(2 k) !}\left|B_{2 k}\right| x^{2 k-1}-\sum_{k=1}^{\infty} \frac{2^{2 k+1}(2 k-1)}{(2 k) !}\left|B_{2 k}\right| x^{2 k-1}+\sum_{k=1}^{\infty} \frac{(2 k-1)\left(2^{2 k}-1\right)}{(2 k) !}\left|B_{2 k}\right| x^{2 k-1}}{\sum_{k=1}^{\infty} \frac{3 \cdot 2^{2 k}(2 k-1)}{(2 k) !}\left|B_{2 k}\right| x^{2 k+1}-\sum_{k=1}^{\infty} \frac{3 \cdot 2^{2}\left(2^{2 k-1}-1\right)}{(2 k) !}\left|B_{2 k}\right| x^{2 k+1}}\right) \\
& =\frac{\sum_{k=1}^{\infty} 2\left[3\left(2^{2 k-1}-1\right)-2^{2 k}(2 k-1)+(2 k-1)\left(2^{2 k-1}-1\right)\right] \frac{\left|B_{2 k}\right|}{(2 k) !} x^{2 k-1}}{\sum_{k=1}^{\infty} 12\left[(2 k-1) 2^{2 k-2}-\left(2^{2 k-1}-1\right)\right] \frac{\left|B_{2 k}\right|}{(2 k) !} x^{2 k+1}} \\
& =\frac{\sum_{k=2}^{\infty} 2\left[3\left(2^{2 k+1}-1\right)-2^{2 k+2}(2 k+1)+(2 k+1)\left(2^{2 k+1}-1\right)\right] \frac{\left|B_{2 k+2}\right|}{(2 k+2) !} x^{2 k+1}}{\sum_{k=1}^{\infty} 12\left[(2 k-1) 2^{2 k-2}-\left(2^{2 k-1}-1\right)\right] \frac{\left|B_{2 k}\right|}{(2 k) !} x^{2 k+1}} \\
& =\frac{\sum_{k=2}^{\infty} 4\left[-2 k \cdot 2^{2 k}+2 \cdot 2^{2 k}-k-2\right] \frac{\left|B_{2 k+2}\right|}{(2 k+2) !} x^{2 k+1}}{\sum_{k=2}^{\infty} 3\left[2 k \cdot 2^{2 k}-3 \cdot 2^{2 k}+4\right] \frac{\left|B_{2 k}\right|}{(2 k) !} x^{2 k+1}} \\
& =\left(\frac{-1}{\sum_{k=2}^{\infty} \frac{180\left|B_{2 k}\right|}{(2 k) !}\left(2 k \cdot 2^{2 k}-3 \cdot 2^{2 k}+4\right) x^{2 k-2}}:=M(x)\right) \\
& -\left(\frac{\sum_{k=2}^{\infty} \frac{4\left|B_{2 k+2}\right|}{(2 k+2) !}\left(2 k \cdot 2^{2 k}-2 \cdot 2^{2 k}+k+2\right) x^{2 k+1}}{\sum_{k=2}^{\infty} \frac{3\left|B_{2 k}\right|}{(2 k) !}\left(2 k \cdot 2^{2 k}-3 \cdot 2^{2 k}+4\right) x^{2 k+1}}:=\frac{A(x)}{B(x)}\right) .
\end{aligned}
$$

Now

$$
2 k \cdot 2^{2 k}-2 \cdot 2^{2 k}+k+2>0,(k=2,3,4, \cdots),
$$

and

$$
2 k \cdot 2^{2 k}-3 \cdot 2^{2 k}+4>0,(k=2,3,4, \cdots) .
$$

Therefore, $M(x)$ is strictly increasing on $(0, \pi / 2)$. Next, let us notice that

$$
\frac{A(x)}{B(x)}=\frac{\sum_{k=2}^{\infty} a_{k} x^{2 k+1}}{\sum_{k=2}^{\infty} b_{k} x^{2 k+1}}
$$

where

$$
a_{k}:=\frac{4\left|B_{2 k+2}\right|}{(2 k+2) !}\left(2 k \cdot 2^{2 k}-2 \cdot 2^{2 k}+k+2\right)>0
$$


and

$$
b_{k}:=\frac{3\left|B_{2 k}\right|}{(2 k) !}\left(2 k \cdot 2^{2 k}-3 \cdot 2^{2 k}+4\right)>0 .
$$

So,

$$
\frac{a_{k}}{b_{k}}=\frac{2}{3} \frac{\left|B_{2 k+2}\right|}{\left|B_{2 k}\right|} \frac{1}{(k+1)(2 k+1)} \frac{\left(2 k \cdot 2^{2 k}-2 \cdot 2^{2 k}+k+2\right)}{\left(2 k \cdot 2^{2 k}-3 \cdot 2^{2 k}+4\right)}:=c_{k}
$$

and

Hence

$$
c_{k+1}=\frac{1}{6} \frac{\left|B_{2 k+4}\right|}{\left|B_{2 k+2}\right|} \frac{1}{(k+2)(2 k+3)} \frac{\left(8 k \cdot 2^{2 k}+k+3\right)}{\left(2 k \cdot 2^{2 k}-2^{2 k}+1\right)} .
$$

$$
\begin{aligned}
\frac{c_{k+1}}{c_{k}}=\frac{1}{4} \frac{\left|B_{2 k+4}\right|}{\left|B_{2 k+2}\right|} & \frac{\left|B_{2 k}\right|}{\left|B_{2 k+2}\right|} \frac{(k+1)(2 k+1)}{(k+2)(2 k+3)} \\
& \times \frac{\left(8 k \cdot 2^{2 k}+k+3\right)\left(2 k \cdot 2^{2 k}-3 \cdot 2^{2 k}+4\right)}{\left(2 k \cdot 2^{2 k}-2^{2 k}+1\right)\left(2 k \cdot 2^{2 k}-2 \cdot 2^{2 k}+k+2\right)} .
\end{aligned}
$$

By Lemma 4, we have

$$
\frac{\left|B_{2 k+4}\right|}{\left|B_{2 k+2}\right|}<\frac{2\left(4 \cdot 2^{2 k}-1\right)(k+2)(2 k+3)}{\pi^{2}\left(16 \cdot 2^{2 k}-1\right)}
$$

and

Then

$$
\frac{\left|B_{2 k}\right|}{\left|B_{2 k+2}\right|}<\frac{\pi^{2}\left(2 \cdot\left(2^{2 k}-1\right)\right.}{\left(2^{2 k}-2\right)(k+1)(2 k+1)} .
$$

$$
\begin{aligned}
4 \frac{c_{k+1}}{c_{k}}< & \frac{2\left(4 \cdot 2^{2 k}-1\right)\left(2 \cdot 2^{2 k}-1\right)}{\left(16 \cdot 2^{2 k}-1\right)\left(2^{2 k}-2\right)} \\
& \times \frac{\left(8 k \cdot 2^{2 k}+k+3\right)\left(2 k \cdot 2^{2 k}-3 \cdot 2^{2 k}+4\right)}{\left(2 k \cdot 2^{2 k}-2^{2 k}+1\right)\left(2 k \cdot 2^{2 k}-2 \cdot 2^{2 k}+k+2\right)}:=N(k) .
\end{aligned}
$$

We claim that

$$
N(k)<4,
$$

i.e.,

$$
\begin{aligned}
& 2\left(4 \cdot 2^{2 k}-1\right)\left(2 \cdot 2^{2 k}-1\right)\left(8 k \cdot 2^{2 k}+k+3\right)\left(2 k \cdot 2^{2 k}-3 \cdot 2^{2 k}+4\right) \\
& \quad<4\left(16 \cdot 2^{2 k}-1\right)\left(2^{2 k}-2\right)\left(2 k \cdot 2^{2 k}-2^{2 k}+1\right)\left(2 k \cdot 2^{2 k}-2 \cdot 2^{2 k}+k+2\right)
\end{aligned}
$$

or

$$
\begin{aligned}
& \left(8 \cdot 2^{4 k}-6 \cdot 2^{2 k}+1\right) \\
& \times\left(16 k^{2} \cdot 2^{4 k}-24 k \cdot 2^{4 k}+2 k^{2} \cdot 2^{2 k}+35 k \cdot 2^{2 k}-9 \cdot 2^{2 k}+4 k+12\right) \\
& <\left(32 \cdot 2^{4 k}-66 \cdot 2^{2 k}+4\right) \\
& \left(4 k^{2} \cdot 2^{4 k}-6 k \cdot 2^{4 k}+2 \cdot 2^{4 k}+2 k^{2} \cdot 2^{2 k}+5 k \cdot 2^{2 k}-4 \cdot 2^{2 k}+k+2\right) .
\end{aligned}
$$


Equivalently, after some computations, we get

$$
\begin{gathered}
\left(120 k^{2}+188\right) \cdot 2^{6 k}+\left(120 k^{2}+120\right) \cdot 2^{4 k}+\left[(57 k+67) \cdot 2^{2 k}+4\right] \\
<\left(64 \cdot 2^{2 k}\right) \cdot 2^{6 k}+\left(132 k \cdot 2^{2 k}\right) \cdot 2^{4 k}+\left[\left(186 \cdot 2^{2 k}+6 k^{2}\right) \cdot 2^{2 k}\right]
\end{gathered}
$$

which is clearly true for $k=2,3,4, \cdots$. This implies that $c_{k+1}<c_{k}$, i.e., a sequence $\left\{a_{k} / b_{k}\right\}_{k=2}^{\infty}$ is strictly decreasing. By Lemma $3, A(x) / B(x)$ is strictly decreasing on $(0, \pi / 2)$ giving us that $F(x)$ is strictly increasing on $(0, \pi / 2)$. Consequently, for $x<\pi / 2$,

$$
F(x)<F\left(\frac{\pi}{2}-\right)=\left(\frac{2}{\pi}-\frac{2}{3}\right)\left(\frac{\pi}{2}-1\right)^{-2}
$$

and the desired right inequality of (2.1) follows.

Remark 1. Fortunately, we can give a very simple proof of the right inequality of (2.1). Indeed, we can write $F(x)$ as follows:

$$
F(x)=\frac{1}{3} \cdot Q(x) \cdot R(x) \cdot[T(x)]^{2},
$$

where

$$
Q(x):=\frac{3 \sin x-2 x-x \cos x}{x^{5}}, \quad R(x):=\frac{1}{\sin ^{2} x}, \quad T(x):=\frac{x^{2} \sin x}{x-\sin x} .
$$

As $R(x)$ is clearly positive and strictly decreasing on $(0, \pi / 2)$, we conclude by Lemmas 5 and 6 that $F(x)$ is strictly increasing on $(0, \pi / 2)$ and the required inequality follows.

Proof of Theorem 2. For the left inequality of (2.2), let us set

$$
g(x):=\frac{\frac{\sin x}{x}-\frac{2+\cos x}{3}}{\sin x-x \cos x}=\frac{3 \sin x-x \cos x-2 x}{3 x \sin x-3 x^{2} \cos x}, \quad x \in(0, \pi / 2) .
$$

We now introduce the following intermediary functions:

$$
\begin{gathered}
g_{1}(x):=3 \sin x-x \cos x-2 x, h_{1}(x):=3 x \sin x-3 x^{2} \cos x, \\
g_{2}(x):=x \sin x+2 \cos x-2, \quad h_{2}(x):=3 \sin x-3 x \cos x+3 x^{2} \sin x,
\end{gathered}
$$

and

$$
g_{3}(x):=x \cos x-\sin x, \quad h_{3}(x):=9 x \sin x+3 x^{2} \cos x .
$$

Then

$$
g_{i}(0+)=h_{i}(0+)=0(i=1,2,3), \quad \frac{g_{i}^{\prime}(x)}{h_{i}^{\prime}(x)}=\frac{g_{i+1}(x)}{h_{i+1}(x)}(i=1,2)
$$

and

$$
\frac{g_{3}^{\prime}(x)}{h_{3}^{\prime}(x)}=\frac{-x \sin x}{9 \sin x+15 x \cos x-3 x^{2} \sin x}=-\frac{x}{3 v(x)}
$$


with

$$
v(x):=\frac{5 x}{\tan x}-x^{2}+3 .
$$

The function $v(x)$ is positive on $(0, \pi / 2)$ because of the obvious relation $\tan x / x>$ $5 /\left(x^{2}-3\right)$ and $v(x)$ is also strictly decreasing on $(0, \pi / 2)$. Applying Lemma 1 repeatedly we get $g(x)$ strictly decreasing on $(0, \pi / 2)$. Hence, for $x<\pi / 2$,

$$
g(x)>g\left(\frac{\pi}{2}-\right)=\frac{2}{\pi}-\frac{2}{3},
$$

giving the left inequality of (2.2).

For the right inequality of $(2.2)$, consider

$$
G(x):=\frac{\frac{\sin x}{x}-\frac{2+\cos x}{3}}{(\sin x-x \cos x)^{2}}=\frac{1}{3} \cdot[P(x)]^{2} \cdot Q(x) \cdot R(x),
$$

where

$$
P(x):=\frac{x^{2} \sin x}{\sin x-x \cos x}, \quad Q(x):=\frac{3 \sin x-2 x-x \cos x}{x^{5}}, \quad R(x):=\frac{1}{\sin ^{2} x} .
$$

By Lemmas 2 and 5 , the function $[P(x)]^{2} \cdot Q(x)$ is negative increasing and $R(x)$ is positive decreasing. Therefore, $G(x)$ is strictly increasing on $(0, \pi / 2)$ and, for $x<\pi / 2$, we have

$$
G(x)<G\left(\frac{\pi}{2}-\right)=\frac{2}{\pi}-\frac{2}{3} .
$$

This completes the proof.

\section{Complements}

This section discusses the importance of our results, by comparing the obtained bounds through analytical and graphical approaches, and also providing alternative proofs.

4.1. Comparison of the obtained bound. The bounds obtained in Theorems 1 and 2 are comparable. Indeed, we claim that

- the lower bound of Theorem 1 is better to the one of Theorem 2,

- the upper bound of Theorem 2 is better to the one of Theorem 1 ,

which is strictly equivalent to say that $\Phi_{1}(x)<\Psi_{1}(x)$ for $x \in(0, \pi / 2)$. After basics developments, we arrive at that is

$$
\Phi_{1}(x)<\Psi_{1}(x) \Leftrightarrow \frac{2}{\pi}+\left(1-\frac{2}{\pi}\right) \cos (x)<\frac{\sin x}{x},
$$

which is an inequality proved by [5]. Finally, a best of Theorems 1 and 2 is the following inequality: 


$$
\frac{2+\cos x}{3}-\left(\frac{2}{3}-\frac{2}{\pi}\right) \Phi_{1}(x)<\frac{\sin x}{x}<\frac{2+\cos x}{3}-\left(\frac{2}{3}-\frac{2}{\pi}\right) \Psi_{2}(x) .
$$

4.2. Graphical analysis. We now provide a graphical analysis of the bounds in Theorems 1 and 2, by distinguishing lower bounds and upper bounds. Figure 1 presents the curves of the lower bounds of the two theorems, both minus $\sin x / x$ for visual comfort.

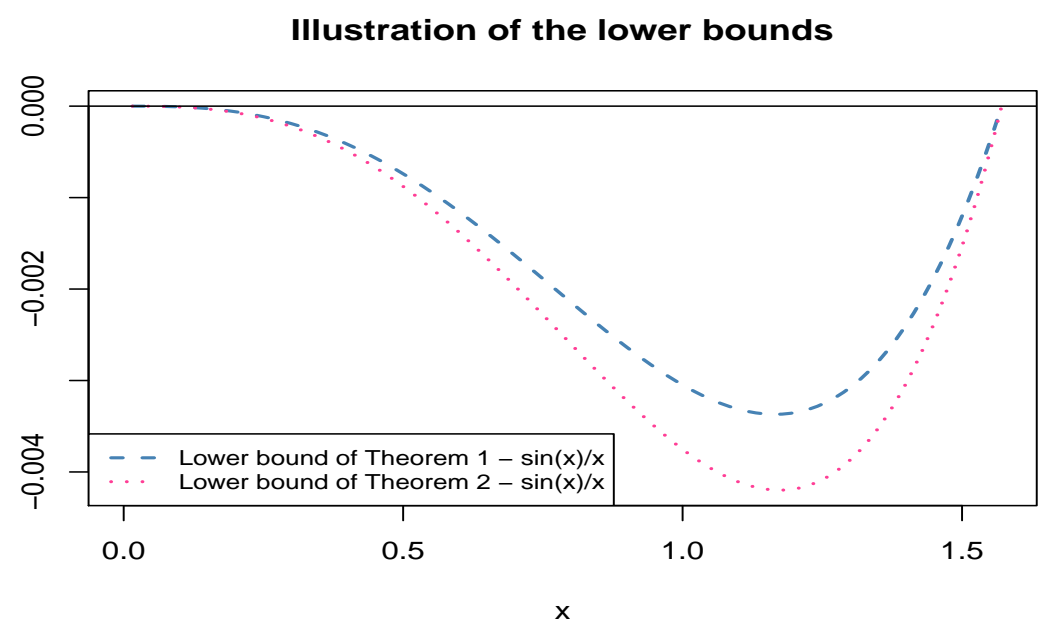

Figure 1. Plots of "lower bounds of Theorems 1 and $2-\sin x / x$ "

Two immediate remarks come from Figure 1. First, the obtained lower bounds are very sharp; the worst magnitude of the worst of the two curves being a remarkable $\approx 0.004$. As a second remark, it is clear that the lower bound of Theorem 1 is uniformly the best, as proved previously.

Figure 2 displays the curves of the upper bounds of the two theorems, both minus $\sin x / x$. 


\section{Illustration of the upper bounds}

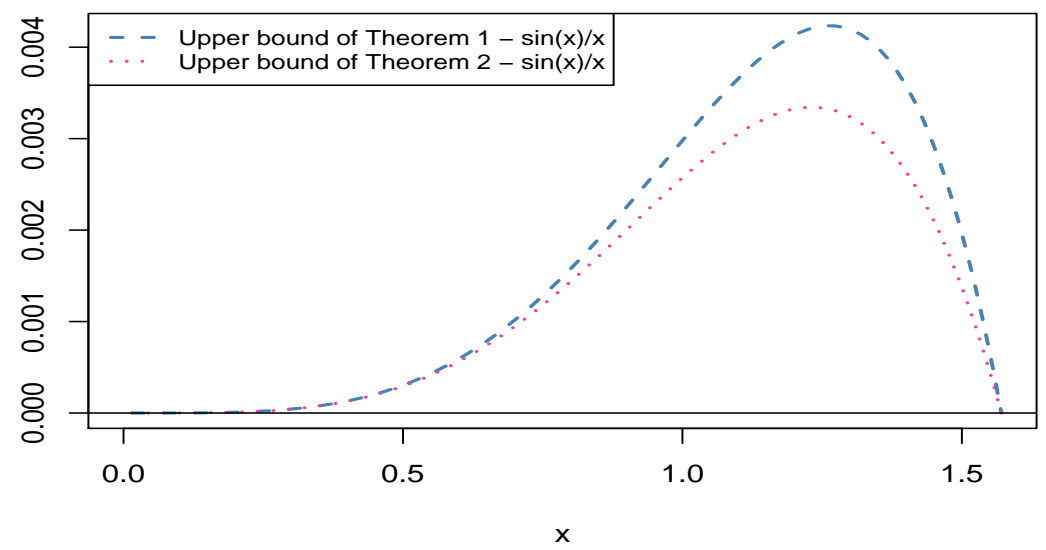

Figure 2. Plots of "upper bounds of Theorems 1 and $2-\sin x / x$ "

Figure 2 illustrates the sharpness of the obtained upper bounds; the worst magnitude of the worst of the two curves being $\approx 0.004$. It also shows that the upper bound of Theorem 2 is uniformly the best, as discussed previously.

4.3. Alternative proofs of main results. It is worth mentioning that alternative proofs of Theorem 1 and 2 can be developed. Such an alternative is proposed below, based on the methodology developed by [23] and [24]. First, let us notice that the functions

$$
\Phi_{1}(x)=\frac{x-\sin x}{\pi / 2-1}, \quad \Phi_{2}(x)=\left(\frac{x-\sin x}{\pi / 2-1}\right)^{2}
$$

and

$$
\Psi_{1}(x)=\sin x-x \cos x, \quad \Psi_{2}(x)=(\sin x-x \cos x)^{2}
$$

involved in Theorems 1 and 2 are Mixed Trigonometric Polynomial (MTP) functions in regards to [23] and [24]. Also, our main results can be formulated as the following inequality:

$$
\frac{2+\cos x}{3}-a \cdot \varphi(x)<\frac{\sin x}{x} x<\frac{2+\cos x}{3}-a \cdot \psi(x),
$$

where $a=2 / 3-2 / \pi, \varphi \in\left\{\Phi_{1}, \Psi_{1}\right\}$ and $\psi \in\left\{\Phi_{2}, \Psi_{2}\right\}$ for $x \in(0, \pi / 2)$. Therefore, the previous inequality can be written under the form of an equivalent double sided inequality as

$$
-\varphi(x)<\frac{1}{a}\left(\frac{\sin x}{x} x-\frac{2+\cos x}{3}\right)<-\psi(x),
$$


for $x \in(0, \pi / 2)$. Since $\varphi(x)$ and $\psi(x)$ are MTP functions, then the problem of proving the previous double sided inequality can be considered by using methods presented in [23] and [24]. Here, is shown how the considered inequalities in Theorem 1 and 2 can be reduced to corresponding MTP inequalities.

- Case 1: $\varphi(x)=\Phi_{1}(x)=(x-\sin x) /(\pi / 2-1)$. In this case, for $x \in$ $(0, \pi / 2)$, we have

$$
\begin{aligned}
& \frac{1}{a}\left(\frac{\sin x}{x} x-\frac{2+\cos x}{3}\right)+\varphi(x)>0 \\
& \Leftrightarrow \frac{3 \pi}{2 \pi-6}\left(\frac{\sin x}{x} x-\frac{2+\cos x}{3}\right)+\frac{x-\sin x}{\pi / 2-1}>0 .
\end{aligned}
$$

This is equivalent to prove that $f_{1}(x)>0$, where

$$
\begin{aligned}
f_{1}(x) & :=\left((-4 \pi+12) x+3 \pi^{2}-6 \pi\right) \sin x \\
& -\pi(\pi-2) x \cos x+x\left((4 \pi-12) x-2 \pi^{2}+4 \pi\right) .
\end{aligned}
$$

Or $f_{1}(x)>0$ is a MTP inequality which can be proved using methods from [23] and [24]. For example, let us state proof in short form according to [24]. Let us set

$$
P_{1}(x):=(-4 \pi+12) x+3 \pi^{2}-6 \pi, \quad P_{2}(x):=-\pi(\pi-2) x
$$

and

$$
P_{3}(x):=x\left((4 \pi-12) x-2 \pi^{2}+4 \pi\right) .
$$

Let also $T_{n}^{f, 0}(x)$ be TAYLOR's polynomial function $f(x)$ of $n^{\text {th }}$ degree at the point $a=0$. Then, it is possible to check that from one side it is true that 


$$
\begin{aligned}
f_{1}(x) & >\underbrace{P_{1}(x)}_{(>0)} T_{7}^{\sin , 0}(x)+\underbrace{P_{2}(x)}_{(<0)} T_{4}^{\cos , 0}(x)+P_{3}(x) \\
& =\left(\frac{\pi}{1260}-\frac{1}{480}\right) x^{8}+\left(-\frac{\pi^{2}}{1680}+\frac{1}{840}\right) x^{7} \\
& +\left(-\frac{\pi}{30}+\frac{1}{100}\right) x^{6}+\left(-\frac{\pi^{2}}{60}+\frac{\pi}{30}\right) x^{5}+\left(\frac{2 \pi}{3}-2\right) x^{4} \\
& =1.12375 \ldots \cdot 10^{-4} x^{8}-2.13477 \ldots \cdot 10^{-3} x^{7} \\
& -4.71975 \ldots \cdot 10^{-3} x^{6}-5.97736 \ldots \cdot 10^{-2} x^{5}+9.43951 \ldots \cdot 10^{-2} x^{4}> \\
& >1.12 \cdot 10^{-4} x^{8}-2.14 \cdot 10^{-3} x^{7} \\
& -4.72 \cdot 10^{-3} x^{6}-5.98 \cdot 10^{-2} x^{5}+9.43 \cdot 10^{-2} x^{4} \\
& =\frac{7}{62500} x^{8}-\frac{107}{50000} x^{7}-\frac{59}{12500} x^{6}-\frac{299}{5000} x^{5}+\frac{943}{10000} x^{4}>0
\end{aligned}
$$

for $x \in(0,1.35)$, and that, from other side, it is true that

$$
\begin{aligned}
f_{1}\left(\frac{\pi}{2}-x\right) & >\underbrace{P_{1}\left(\frac{\pi}{2}-x\right)}_{(>0)} T_{2}^{\cos , 0}(x)+\underbrace{P_{2}\left(\frac{\pi}{2}-x\right)}_{(<0)} T_{1}^{\sin , 0}(x)+P_{3}\left(\frac{\pi}{2}-x\right) \\
& =(-2 \pi+6) x^{3}+\left(\pi^{2} / 2+2 \pi-12\right) x^{2}+\left(-\pi^{3} / 2-\pi^{2}+12 \pi-12\right) x \\
& =-2.83185 \ldots \cdot 10^{-1} x^{3}-7.82012 \ldots \cdot 10^{-1} x^{2}+3.26369 \ldots \cdot 10^{-1} x \\
& >-2.84 \cdot 10^{-1} x^{3}-7.83 \cdot 10^{-1} x^{2}+3.26 \cdot 10^{-1} x \\
& =-\frac{71}{250} x^{3}-\frac{783}{1000} x^{2}+\frac{163}{500} x>0
\end{aligned}
$$

for $x \in(0,0.36)$.

Thus, the MTP inequality $f_{1}(x)>0$ for $x \in(0, \pi / 2)$ is wholesomely proved. The other cases are mentioned below, with less details. 
- Case 2: $\psi(x)=\Phi_{2}(x)=((x-\sin x) /(\pi / 2-1))^{2}$. In this case, for $x \in(0, \pi / 2)$, we have

$$
\begin{aligned}
& \frac{1}{a}\left(\frac{\sin x}{x} x-\frac{2+\cos x}{3}\right)+\psi(x)<0 \\
& \Leftrightarrow \frac{3 \pi}{2 \pi-6}\left(\frac{\sin x}{x} x-\frac{2+\cos x}{3}\right)+\left(\frac{x-\sin x}{\pi / 2-1}\right)^{2}<0 \\
& \Leftrightarrow f_{2}(x):=-8 x(\pi-3) \cos ^{2} x-\pi x(\pi-2)^{2} \cos x \\
& +\left((-16 \pi+48) x^{2}+3 \pi(\pi-2)^{2}\right) \sin x \\
& -2 \pi^{3} x+8 \pi x^{3}+8 \pi^{2} x-24 x^{3}-24 x<0 .
\end{aligned}
$$

The MTP inequality $f_{2}(x)<0$ over $(0, \pi / 2)$ can be proven using methods from [23] and [24].

- Case 3: $\varphi(x)=\Psi_{1}(x)=\sin x-x \cos x$. In this case, for $x \in(0, \pi / 2)$, it holds that

$$
\begin{aligned}
& \frac{1}{a}\left(\frac{\sin x}{x} x-\frac{2+\cos x}{3}\right)+\varphi(x)>0 \\
& \Leftrightarrow \frac{3 \pi}{2 \pi-6}\left(\frac{\sin x}{x} x-\frac{2+\cos x}{3}\right)+\sin x-x \cos x>0 \\
& \Leftrightarrow f_{3}(x):=(2(\pi-3)+3 \pi) \sin x+x(-2(\pi-3) x-\pi) \cos x-2 \pi x>0 .
\end{aligned}
$$

Hence, the MTP inequality $f_{3}(x)>0$ over $(0, \pi / 2)$ can be proved via the methods from [23] and [24].

- Case 4: $\psi(x)=\Psi_{2}(x)=(\sin x-x \cos x)^{2}$. For $x \in(0, \pi / 2)$, we have

$$
\begin{aligned}
& \frac{1}{a}\left(\frac{\sin x}{x} x-\frac{2+\cos x}{3}\right)+\psi(x)<0 \\
& \Leftrightarrow \frac{3 \pi}{2 \pi-6}\left(\frac{\sin x}{x} x-\frac{2+\cos x}{3}\right)+(\sin x-x \cos x)^{2}<0 \\
& \Leftrightarrow f_{4}(x):=-4 x^{2}(\pi-3) \sin x \cos x+3 \pi \sin x \\
& +2 x\left(x^{2}-1\right)(\pi-3) \cos ^{2} x-\pi x \cos x-6 x<0 .
\end{aligned}
$$

And, the MTP inequality $f_{4}(x)<0$ over $(0, \pi / 2)$ can be proven using methods from [23] and [24].

This ends this possible alternative proof.

\section{REFERENCES}

[1] H. Alzer, and S. L. Qiu, Monotonicity theorems and inequalities for the complete elliptic integrals, J. Comput. Appl. Math., 172 (2004), 289-312.

[2] G. D. Anderson, M. K. Vamanamurthy and M. Vuorinen, Monotonicity rules in calculus, Amer. Math. Monthly. 133(9) (2006), 805-816. 
[3] Y. J. Bagul, Remark on the paper of Zheng Jie Sun and Ling Zhu, J. Math. Ineqal. 13(3) (2019), 801-803.

[4] Y. J. Bagul, and C. Chesneau, Some sharp circular and hyperbolic bounds of $\exp \left(-x^{2}\right)$ with applications, Applicable Analysis and Discrete Mathematics, 14(1) (2020), 239254. Doi: 10.2298/AADM190123010B

[5] Y. J. Bagul and C. Chesneau, Refined forms of Oppenheim and Cusa-Huygens type inequalities, Acta Et Commentationes Universitatis Tartueensis De Mathematica (Accepted for Publication).

[6] Y. J. Bagul, C. Chesneau, M. Kostić, On the Cusa-Huygens inequality, Preprint, (2020), (hal-02475321).

[7] Y. J. Bagul, C. Chesneau, M. Kostić, The Cusa-Huygens inequality revisited, Novi Sad Journal of Mathematics (Accepted for publication). Doi: 10.30755/NSJOM.10667

[8] C.-P. Chen and W.-S. Cheung, Sharp Cusa and Becker-Stark inequalities, J. Inequal. Appl., vol. 2011, Article no. 136, 2011.

[9] C.-P. Chen, J. Sándor, Inequality chains for Wilker, Huygens and Lazarević type inequalities, J. Math. Inequal. 8 (2014), 55-67.

[10] R. M. Dhaigude, C. Chesneau and Y. J. Bagul, About trigonometric-polynomial bounds of sinc function, Math. Sci. Appl. E-Notes, 8(1) (2020), 100-104.

[11] I. S. Gradshteyn and I. M. Ryzhik, Table of Integrals, Series and Products, Elsevier, edn. 2007.

[12] V. Heikkala, M. K. Vamanamurthy, and M. Vuorinen, Generalized elliptic integrals, Comput. Methods Funct. Theory, 9(1) (2009), 75-109.

[13] C. Huygens, Oeuvres Completes, Société Hollandaise des Sciences, Haga, 1888-1940.

[14] B. Malešević, M. Nenezić, L. Zhu, B. Banjac and M. Petrović, Some new estimates of precision of Cusa-Huygens and Huygens approximations, preprint, 2019. https://arxiv.org/abs/1907.00712.

[15] B. Malešević, T. Lutovac, M. Rašajski and C. Mortici, Extensions of the natural approach to refinements and generalizations of some trigonometric inequalities, Advances in Difference Equations, 2018, 2018:90.

[16] D. S. Mitrinović, Analytic Inequalities, Springer-Verlag, Berlin, 1970.

[17] C. Mortici, The natural approach of Wilker-Cusa-Huygens Inequalities, Math. Inequal. Appl. 14(3) (2011), 535-541.

[18] E. Neuman and J. Sándor, On some inequalities involving trigonometric and hyperbolic functions with emphasis on the Cusa-Huygens, Wilker and Huygens inequalities, Math. Inequal. Appl. 13(4) (2010), 715-723.

[19] F. Qi, A double inequality for the ratio of two non-zero neighbouring Bernoulli numbers, Journal of Computational and Applied Mathematics, 351 (2019), 1-5.

[20] J. Sándor, Sharp Cusa-Huygens and related inequalities, Notes Number Theory Discrete Math. 19 (2013), 50-54.

[21] J. Sándor and R. Oláh-Gal, On Cusa-Huygens type trigonometric and hyperbolic inequalities, Acta. Univ. Sapientiae Mathematica 4(2) (2012), 145-153.

[22] L. Zhu, New Cusa-Huygens type inequalities, AIMS Mathematics, 5(5) (2020), 53205331. Doi: $10.3934 /$ math.2020341

[23] B. Malešević, M. Makragić, A Method for Proving Some Inequalities on Mixed Trigonometric Polynomial Functions, J. Math. Inequal. 10:3 (2016), 849-876.

[24] B. Banjac, System for automatic proving of some classes of analytic inequalities, Doctoral dissertation (in Serbian), School of Electrical Engineering, Belgrade, May 2019. Available on: http://nardus.mpn.gov.rs/ 
LMnO, University of Caen-Normandie, Caen, France

E-mail address: christophe.chesneau@unicaen.fr

Faculty of Technical Sciences, University of Novi Sad, Trg D. Obradovića 6, 21125 Novi Sad, Serbia

E-mail address: marco.s@verat.net

School of Electrical Engineering, University of Belgrade, Serbia,

E-mail address: branko.malesevic@etf.bg.ac.rs

Faculty of Technical Sciences, University of Novi Sad, Serbia,

E-mail address: bojan.banjac@uns.ac.rs

Department of Mathematics, K. K. M. College Manwath, Dist: Parbhani(M.S.) - 431505, INDIA

E-mail address: yjbagul@gmail.com 